\title{
Cinétique et caractérisation du phénomène "apoptosis-like" induit au cours de la congélation des spermatozoïdes bovins
}

\author{
Guillaume MARTIN ${ }^{1,2}$, Odile SABIDO ${ }^{3}$, Philippe DURAND ${ }^{2}$, Rachel LEVY $^{1}$ \\ 1 Laboratoire de Biologie de la Reproduction-GIMAP, Hôpital Nord, Saint-Etienne \\ 2 INSERM U418-INRA UMR1245, Hôpital Debrousse, Lyon \\ 3 Centre Commun de Cytométrie en Flux, Université Jean Monnet, Saint-Etienne
}

\section{RESUME}

La congélation des spermatozoïdes éjaculés bovins induit une mortalité cellulaire élevée. Au cours d'une étude préliminaire, nous avions décrit qu'elle s'accompagne d'un processus où apparaissent plusieurs caractéristiques précoces de l'apoptose : i) diminution du potentiel de membrane mitochondrial $\left(\Delta \psi^{\mathrm{m}}\right)$, ii) activation des caspases, iii) augmentation de la perméabilité membranaire, sans apparition des caractéristiques tardives : iv) pas de fragmentation de l'ADN.

Pour préciser ce mécanisme, nous avons étudié séparément l'effet des différentes étapes de la congélation des spermatozoïdes bovins : dilution dans le milieu de congélation, équilibration et congélation dans l'azote liquide/décongélation. L'apoptose et la réaction acrosomique ont été analysées en cytométrie en flux et la présence d'AIF (Apoptosis Inducing Factor) en western blot. Nous avons observé que la dilution dans le milieu de congélation induisait immédiatement une augmentation massive de la proportion de spermatozoïdes avec un $\Delta \psi^{m}$ faible.

Puis, dès l'équilibration, la proportion de spermatozoïdes contenant des caspases actives commence à augmenter. Après le processus complet de congélation/décongélation, cette population est maximale et la perméabilité membranaire apparaît. Ces résultats montrent que le processus "apoptosis-like" est déclenché dès les premières étapes de la congélation. Nous avons aussi mis en évidence la présence d'AIF, ce qui suggère l'implication d'une voie indépendante des caspases, annexe à la voie des caspases.

Mots clés : apoptose, spermatozoïdes bovins, congélation, cytométrie en flux

\section{INTRODUCTION}

La congélation/décongélation des spermatozoïdes induit une diminution de la viabilité des spermatozoïdes bovins [10]. La nécrose et l'apoptose sont deux formes de mort cellulaire différentes. Contrairement à la nécrose qui entraîne une rupture de la membrane plasmique, l'apoptose est une forme de mort cellulaire programmée qui permet une élimination contrôlée et régulée des cellules, sans inflammation [8, 19]. L'apoptose est un phénomène complexe qui peut être divisé de manière schématique en trois phases : induction, exécution et dégradation. Les mitochondries jouent un rôle central pendant la phase d'exécution. Après induction de l'apoptose, les pores mitochondriaux sont ouverts, ce qui se caractérise par une diminution du potentiel de membrane mitochondrial $\left.\Delta \psi^{m}\right)$ [14]. L'ouverture des pores mitochondriaux entraîne le relargage et l'activation de facteurs proapoptotiques dans le cytoplasme. Par exemple, les protéines de la famille des caspases vont être activées, et/ou l'Apoptosis Inducing Factor (AIF) va être relargué des mitochondries [7]. Ces protéines participent ensuite à la phase de dégradation durant laquelle des modifications membranaires et nucléaires sont observées. Les résidus de phosphatidylserine sont externalisés vers le feuillet externe de la membrane plasmique, ce qui facilite la phagocytose des cellules apoptotiques [1], la perméabilité membranaire des cellules s'accroit [5] et l'ADN des cellules apoptotiques est clivé de manière internucléosomale par des endonucléases spécifiques [8].

Au cours d'une précédente étude, nous avions démontré que la congélation des spermatozoïdes bovins induisait

\section{Correspondance :}

Dr Rachel LEVY - Laboratoire de Biologie de la Reproduction, Hôpital Nord, 42055 Saint-Etienne Tel $04 \quad 77828307 \quad$ - Fax $04 \quad 778284 \quad 61 \quad$ Email rachel.levy@chu-st-etienne.fr 
l'apparition d'un processus ressemblant à l'apoptose que nous avons qualifié d'"apoptosis-like" [10]. La congélation des spermatozoïdes bovins s'accompagne ainsi de l'apparition des marqueurs précoces de l'apoptose : i) diminution du $\Delta \psi^{m}$, ii) activation des caspases, iii) augmentation de la perméabilité membranaire. Cependant, la congélation/ décongélation ne s'accompagne pas d'une augmentation de la fragmentation de l'ADN ni de la condensation nucléaire, même de manière tardive (30 heures après décongélation).

Le but de ce travail est de préciser à quel moment de la congélation apparaissent les marqueurs apoptotiques précoces, de préciser les mécanismes impliqués au cours de ce processus et l'effet de la congélation/décongélation des spermatozoïdes bovins sur la réaction acrosomique.

\section{MATERIEL ET METHODES}

\section{Prélèvement et congélation des spermatozoïdes bovins}

Les différentes analyses ont été réalisées à partir de spermatozoïdes prélevés sur dix taureaux de race charolais. Les prélèvements de semence ont été effectués grâce à un vagin artificiel. Après prélèvement, les spermatozoïdes ont été dilués dans le milieu Biociphos (IMV technologies, L'Aigle, France) à $37^{\circ} \mathrm{C}$ à une concentration de 100 millions $/ \mathrm{ml}$ avant d'être mis en paillettes. Les spermatozoïdes sont ensuite équilibrés pendant plus de $3 \mathrm{~h}$ à $4^{\circ} \mathrm{C}$ puis congelés sur les vapeurs d'azote pendant 10 min avant d'être plongés et conservés dans l'azote liquide $[17,18]$. Les spermatozoïdes sont décongelés à $37^{\circ} \mathrm{C}$ pendant $1 \mathrm{~min}$.

\section{Culture et induction de l'apoptose dans les cellules témoins U937}

La lignée leucémique myéloïde humaine de type monocytaire U937 a été utilisée afin de valider les différentes techniques de détection de l'apoptose [16]. Les cellules U937 ont été cultivées dans du milieu RPMI-1640 (Eurobio, Les Ulis, France) contenant de la pénicilline, de la streptomycine et supplémenté par $10 \%$ de sérum de veau fœtal. Les cellules $\mathrm{U} 937$ ont été maintenues dans une atmosphère à $37^{\circ} \mathrm{C}$ avec $5 \%$ de $\mathrm{CO}_{2}$. L'apoptose a été induite par une incubation de $6 \mathrm{~h}$ à $37^{\circ} \mathrm{C}$ avec de l'étoposide (VP-16) (Sigma, Saint Quentin Fallavier, France) à $25 \mu \mathrm{M}$.

\section{Analyse en cytométrie en flux et/ou en microscopie à fluorescence}

Les marqueurs de l'apoptose (diminution du potentiel de membrane mitochondrial $\left(\Delta \psi^{m}\right)$, activation des caspases et augmentation de la perméabilité membranaire) ont été étudiés sur les spermatozoïdes bovins frais après simple dilution dans du PBS, après dilution dans le milieu de congélation Biociphos, après équilibration de $3 \mathrm{~h}$ à $4^{\circ} \mathrm{C}$ et après le processus complet de congélation/décongélation.

Le 3,3'-dihexylocarbocyanine iodide $\left(\mathrm{DiOC}_{6}(3)\right)$ (Molecular Probes, Montluçon, France) a été utilisé à $90 \mathrm{nM}$ final pour détecter les variations du $\Delta \psi^{m}[2,10]$. Le Val-Ala-Asp
Fluoromethylketone couplé au FITC (FITC-VAD-FMK) (Promega, Charbonnière les Bains, France) a été utilisé à $10 \mu \mathrm{M}$ final pour étudier l'activation des caspases [3, 11]. Le Yo-Pro-1 (Molecular Probes) a été utilisé à $100 \mathrm{nM}$ final pour étudier les variations de perméabilité membranaire [5, 10]. La Peanut Agglutinine couplée au FITC (PNA-FITC) (Sigma) a été utilisée à $10 \mu \mathrm{g} / \mathrm{ml}$ pour étudier la réaction acrosomique [13].

Pour tous les marquages, 1 million de cellules ont été diluées au préalable dans $1 \mathrm{ml}$ de PBS. Les cellules ont ensuite été incubées avec l'un des différents marqueurs pendant 20 min à température ambiante.

Avant l'analyse en cytométrie en flux, $6 \mu \mathrm{M}$ d'iodure de propidium (IP) (Sigma) ont été ajoutés à chaque tube afin de déterminer la proportion de cellules nécrotiques. Les analyses ont été réalisées avec le FACS Vantage SE cell-sorter (BD Bioscicences, San Jose, Etats-Unis). Les fluorescences vertes $\left(\mathrm{DiOC}_{6}(3)\right.$, FITC-VAD-FMK, Yo-Pro-1 et PNAFITC) et rouges (IP) ont respectivement été détectées à l'aide des détecteurs FL1 et FL3, à travers des filtres de bandes passantes de 530/30 nm et 695/40 nm. 10000 événements ont été analysés et les fluorescences FL1 et FL3 ont été étudiées après amplification logarithmique grâce au programme Cell Quest Pro 3.1 (BD Biosciences).

Un microscope DMRB (Leica, Wetzlar, Allemagne) équipé d'un filtre L5 (bande passante de $480 / 40 \mathrm{~nm}$ ) a été utilisé pour les analyses de microscopie à fluorescence.

\section{Analyse de l'Apoptosis Inducing Factor (AIF) en Western Blot}

L'analyse en western blot a été réalisée selon une procédure classique. Les spermatozoïdes bovins et les cellules U937 ont été lavés deux fois dans du PBS avant d'être resuspendus dans le tampon de lyse $(50 \mathrm{mM}$ Tris- $\mathrm{HCl}(\mathrm{pH}$ $7,5), 150 \mathrm{mM} \mathrm{NaCl}, 10 \mathrm{mM}$ glycérophosphate, 2 mM EDTA, $2 \mathrm{mM}$ PMSF, $1 \mathrm{mM} \mathrm{Na} 3 \mathrm{VO}_{4}, 1 \mathrm{mM} \mathrm{NaF}, 1 \%$ Nonidet P-40, $1 \mathrm{x}$ inhibiteurs de protéases (Roche, Meylan, France)). Après une incubation sur glace de $10 \mathrm{~min}$ et une étape de centrifugation $\left(13000 \mathrm{~g}, 10 \mathrm{~min}, 4^{\circ} \mathrm{C}\right.$ ) les surnageants ont été prélevés. Les lysats cellulaires ainsi obtenus, équivalents à $25 \mu \mathrm{g}$ de protéines, ont été séparés en utilisant un gel SDS-PAGE à $12 \%$ et transférés sur membrane de PVDF (Bio-Rad, Marnes-la-Coquette, France) en utilisant la procédure standard. Après incubation sur la nuit à $4^{\circ} \mathrm{C}$ dans une solution de saturation ( $8 \%$ de lait en poudre, $0,05 \%$ Tween dans PBS), les membranes ont été incubées avec l'anticorps primaire dilué dans la solution de saturation : anti-AIF, 1/2000 (Sigma) pendant $1 \mathrm{~h}$ à température ambiante. L'anticorps secondaire purifié chez la souris et possédant une forte affinité pour les IgG anti-lapin (Sigma) a été utilisé à $1 / 1000$ dans la solution de saturation à température ambiante. Les bandes spécifiques ont été détectées avec le système ECL (Amersham Biosciences, Orsay, France).

\section{Méthode statistique}

Les analyses statistiques ont été effectuées à l'aide du pro- 
gramme Statistica 6.0 (StatSoft, Tulsa, Etats-Unis). Pour chaque marqueur, 10 expériences ont été réalisées. Les moyennes ont été comparées par le test-t de Student. Les tests-t ont été appariés par animal. Les barres des histogrammes représentent les moyennes +/- écart-types et sont considérées significativement différentes quand $P<0,05$.

\section{RESULTATS}

\section{Différence de potentiel de membrane mitochondrial $\left(\Delta \psi^{\mathrm{m}}\right)$}

L'accumulation du $\mathrm{DiOC}_{6}(3)$ dans la membrane interne des mitochondries permet de détecter les variations du $\Delta \psi^{\mathrm{m}}$. En microscopie à fluorescence, nous avons détecté la fluorescence $\mathrm{DiOC}_{6}(3)$ principalement au niveau de la pièce intermédiaire du spermatozoïde, où sont localisées les mitochondries (Figure 1C). En utilisant le marquage $\mathrm{DiOC}_{6}(3) / \mathrm{IP}$ en cytométrie en flux, trois populations ont été détectées (Figure 1A) : les cellules nécrotiques (a), les cellules vivantes non-apoptotiques avec un $\Delta \psi^{\mathrm{m}}$ normal (c) et les cellules vivantes apoptotiques avec un $\Delta \psi^{m}$ faible (b). Après induction de l'apoptose dans la lignée cellulaire U937, nous avons observé une augmentation de la proportion de cellules vivantes avec $\Delta \psi^{\mathrm{m}}$ faible ; ce qui nous a permis de valider l'expérience.

Nous avons observé que la congélation des spermatozoïdes bovins induit une diminution du $\Delta \psi^{m}$ (Figure 1A) de manière similaire à ce que nous avons observé lors de l'apoptose des cellules U937. Cette diminution du $\Delta \psi^{\mathrm{m}}$ apparaît dès l'étape de dilution des spermatozoïdes dans le milieu de congélation Biociphos (Figure 1B).

\section{Activité des caspases}

En utilisant le marquage FITC-VAD-FMK/IP en cytométrie en flux, trois populations ont été détectées (Figure 2A) : les cellules nécrotiques (a), les cellules vivantes non-apoptotiques sans caspases actives (b) et les cellules vivantes apoptotiques contenant des caspases actives (c). Après induction de l'apoptose dans la lignée cellulaire U937, nous avons observé une augmentation de la proportion de cellules vivantes avec des caspases actives.

Nous avons observé que la congélation des spermatozoïdes bovins induisait aussi une activation des caspases (Figure 2A). De plus, nous avons précisé que le début d'activation des caspases était détectable après équilibration et que le maximum d'activation était obtenu après le processus complet de congélation/décongélation (Figure 2B).

Les caspases actives ont été principalement détectées au niveau de la pièce intermédiaire (Figure $2 \mathrm{C}$ ).

\section{Perméabilité membranaire}

En utilisant le marquage Yo-Pro-1/IP en cytométrie en flux, trois populations ont été détectées (Figure $3 A$ ) : les cellules nécrotiques (a), les cellules vivantes non-apoptotiques imperméable à Yo-Pro-1 (b) et les cellules vivantes apoptotiques perméables au Yo-Pro-1 (c). Après induction de l'a- poptose dans la lignée cellulaire U937, nous avons noté une augmentation de la proportion de cellules vivantes avec une perméabilité membranaire au Yo-Pro-1 accrue.

Nous avons observé que la congélation des spermatozoïdes bovins induisait aussi une augmentation de la perméabilité membranaire au Yo-Pro-1 (Figure $3 \mathrm{~A}$ ) mais seulement après le processus complet de congélation/décongélation (Figure 3B).

\section{Présence d'AIF (Apoptosis Inducing Factor)}

Dans l'étude en western blot des spermatozoïdes bovins avant et après congélation ainsi que des cellules U937 apoptotiques et non apoptotiques, l'anticorps anti-AIF a reconnu une bande majoritaire de $57 \mathrm{kDa}$ correspondant au poids moléculaire de l'AIF (Figure 4).

\section{Réaction acrosomique}

En microscopie à fluorescence, nous avons observé que la fluorescence PNA-FITC est surtout localisée au niveau de l'acrosome (Figure 5B). Le marquage PNA-FITC/IP en cytométrie en flux, nous a permis de détecter trois populations (Figure 5A) : les spermatozoïdes nécrotiques (a), les spermatozoïdes vivants avec un acrosome intact (b) et les spermatozoïdes vivants apoptotiques avec un acrosome réagi (c). Nous avons observé que la congélation des spermatozoïdes bovins induisait une augmentation de la proportion de spermatozoïdes vivants avec un acrosome réagi (Figure $5 A$ et $C)$.

\section{DISCUSSION}

Il est bien établi que le processus de congélation/décongélation a un effet délétère sur les spermatozoïdes [12]. Le pourcentage important de spermatozoïdes présentant une forte perméabilité à l'IP provient d'un processus de nécrose simple ou de nécrose secondaire à l'apoptose.

Comme la présence d'apoptose dans le spermatozoïde éjaculé reste controversée, nous avons dans un premier temps validé chaque test à l'aide de la lignée somatique humaine U937. Après induction de l'apoptose par le VP-16, tous les marqueurs de la cascade d'événements de l'apoptose ont été observés : diminution du $\Delta \psi^{m}$, activation des caspases et augmentation de la perméabilité membranaire au Yo-Pro1. De plus, nous avons mis en évidence la présence d'AlF dans les cellules U937.

Avant congélation, les spermatozoïdes bovins présentent peu de caractéristiques de l'apoptose. Parmi les spermatozoïdes vivants (environ $70 \%$ du nombre total de spermatozoïdes), moins de $10 \%$ ont un faible $\Delta \psi^{m}$, des caspases actives ou une perméabilité importante au Yo-Pro-1. Pendant la spermatogenèse, un grand nombre de cellules germinales est éliminé par apoptose [9]. Parfois, certaines cellules germinales apoptotiques échappent à cette élimination (c'est ce que l'on appelle apoptose abortive) et se retrouvent alors dans l'éjaculat [15]. Selon cette hypothèse, il est possible que les populations apoptotiques basales, que nous avons observées, soient le résultat de cette apoptose abortive. Cependant, comme nous l'avions déterminé 
A

U937
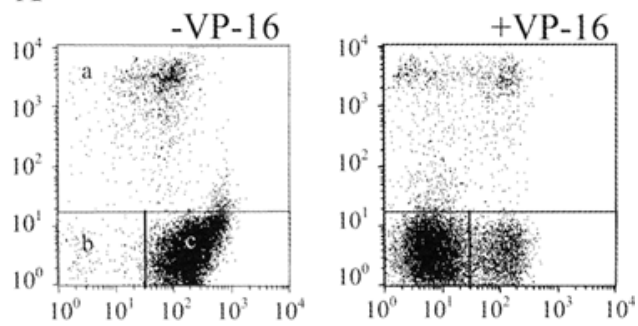

Spermatozoïdes Bovins
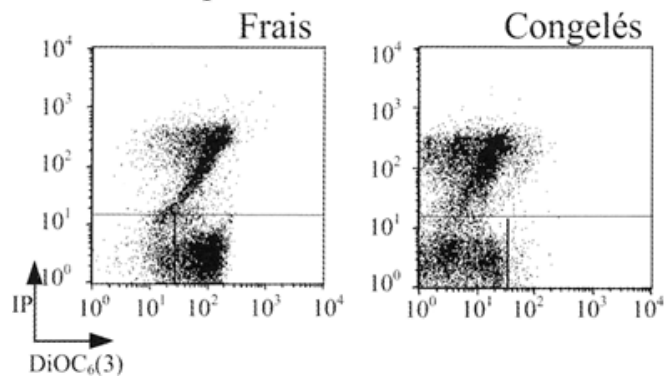

B $\% \operatorname{DiOC}_{6}(3)^{\text {low }} / \mathrm{IP}^{-}$
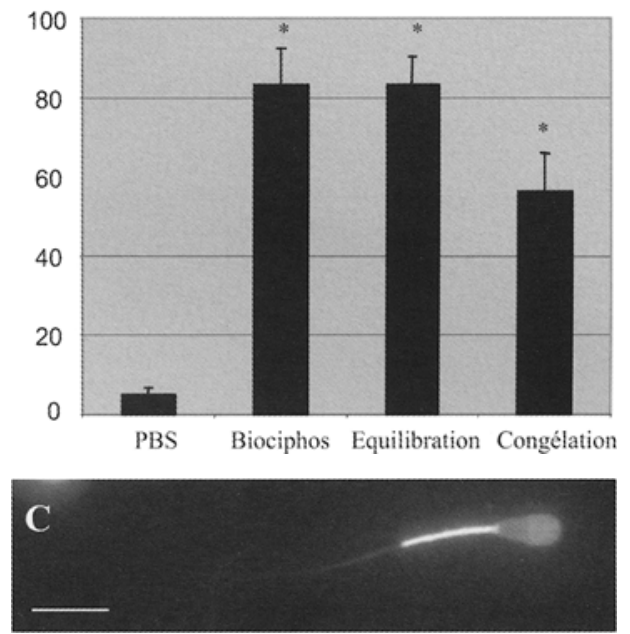

Figure 1 : Etude $d u \Delta \psi_{m}$.

A : Cytogrammes DiOC6(3)/IP. a représente les cellules mortes (IP+), b les cellules apoptotiques (DiOC6(3)/ow/IP-) et $c$ les cellules non-apoptotiques (DiOC6(3)+/IP-). +VP-16 signifie que les cellules U937 ont été traitées par $10 \mu M$ de VP-16 pendant $6 \mathrm{~h}$.

B : Evolution de la proportion de spermatozoïdes vivants apoptotiques (DiOC6(3)low/IP-) au cours du processus de congélation. * signifie que la valeur est différente de celle observée dans le PBS avec $p<0,05$.

$C$ : Localisation du marquage DiOC6(3) dans un spermatozoïde frais, échelle $=5 \mu M$.

A

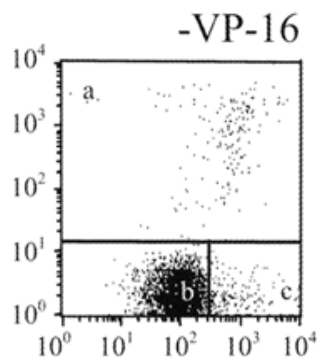

Spermatozoïdes Bovins

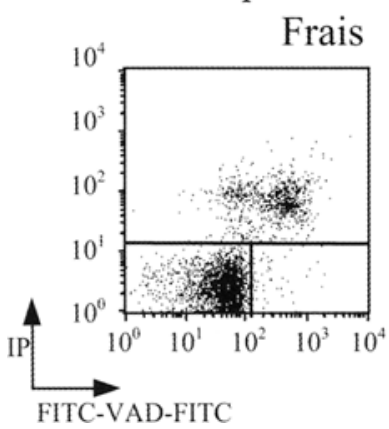

U937
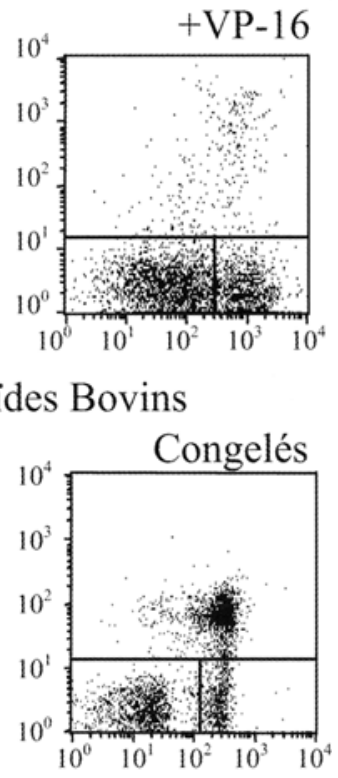

B \% FITC-VAD-FMK ${ }^{+} /$IP $^{-}$
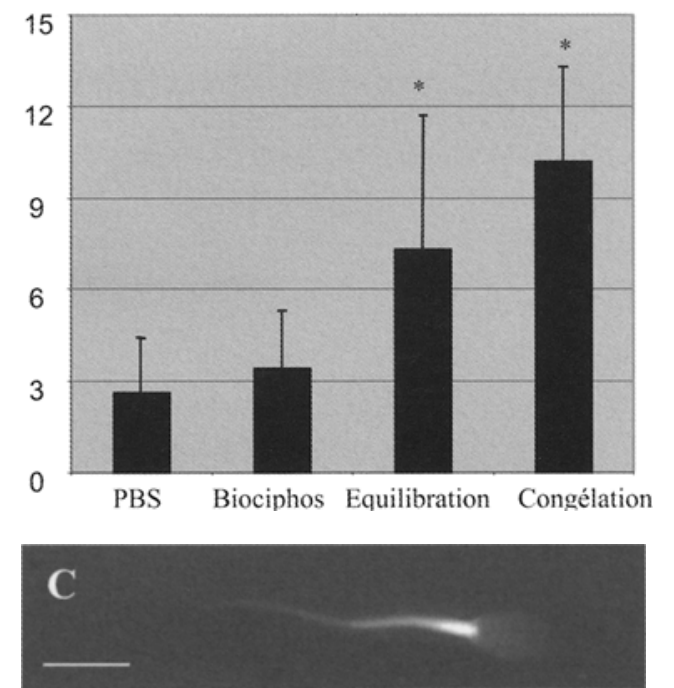

Figure 2 : Etude de l'activation des caspases.

A : Cytogrammes FITC-VAD-FMK/P. a représente les cellules mortes (IP+), b les cellules non-apoptotiques (FITC-VADFMK-IIP-) et $c$ les cellules apoptotiques (FITC-VAD-FMK+/IP-). + VP-16 signifie que les cellules U937 ont été traitées par $10 \mu M$ de VP-16 pendant $6 \mathrm{~h}$.

$B$ : Evolution de la proportion de spermatozoïdes vivants apoptotiques (FITC-VAD-FMK+/IP-) au cours du processus de congélation. * signifie que la valeur est différente de celle observée dans le PBS avec $p<0,05$.

$C$ : Localisation du marquage FITC-VAD-FMK dans un spermatozoïde congelé, échelle $=5 \mu M$. 
A

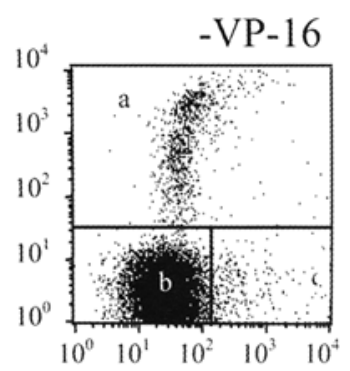

Spermatozoïdes Bovins

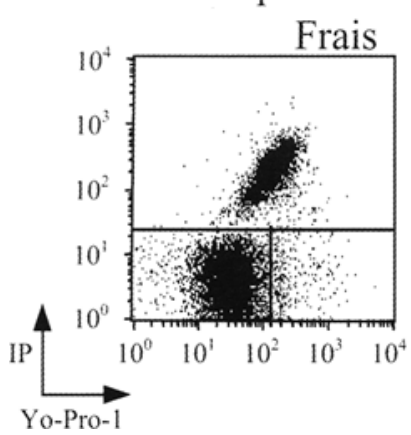

U937
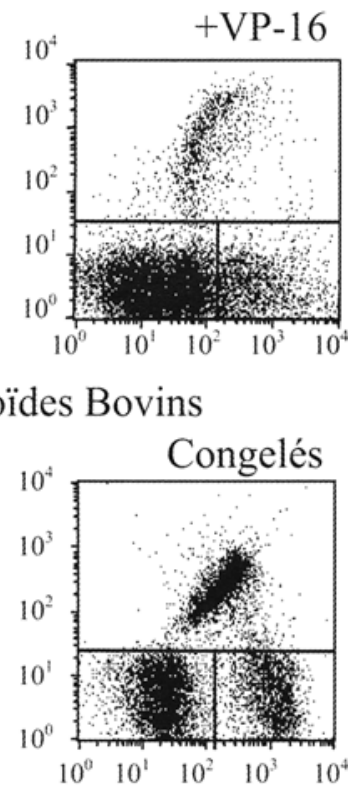

B $\%$ Yo-Pro- $1^{+} /$IP $^{-}$

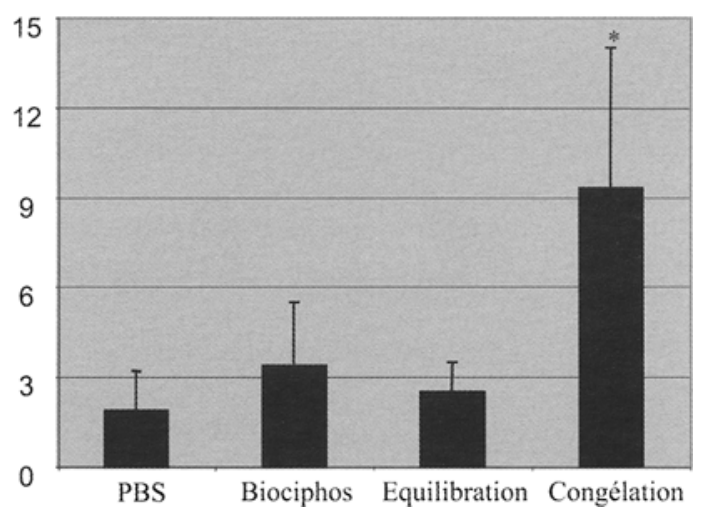

Figure 3 : Etude de l'activation de la perméabilité membranaire.

$A$ : Cytogrammes Yo-Pro-1/IP. a représente les cellules mortes (IP+), b les cellules non-apoptotiques (Yo-Pro-1-/IP-) et $C$ les cellules apoptotiques (Yo-Pro-1+/IP-). + VP-16 signifie que les cellules U937 ont été traitées par $10 \mu M$ de VP-16 pendant $6 h$.

B : Evolution de la proportion de spermatozoïdes vivants apoptotiques (Yo-Pro-1+/IP-) au cours du processus de congélation. * signifie que la valeur est différente de celle observée dans le PBS avec $p<0,05$.

Taureau 1 Taureau 2 Taureau $3 \quad$ U937

F $\quad \mathrm{C} \quad \mathrm{F} \quad \mathrm{C} \quad \mathrm{F} \quad \mathrm{C}-{ }_{-}$

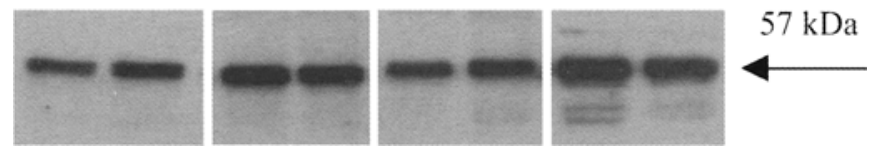

Figure 4 : Etude de la présence d'AlF en western blot.

$F$ représente les spermatozoïdes bovins frais et $C$ congelés. Les 3 western blots présentés sont des exemples représentatifs des western blots réalisés pour les 10 taureaux. - signifie que les cellules U937 n'ont pas été traitées et + qu'elles ont été traitées par $10 \mu M$ de VP-16 pendant $6 \mathrm{~h}$.
A

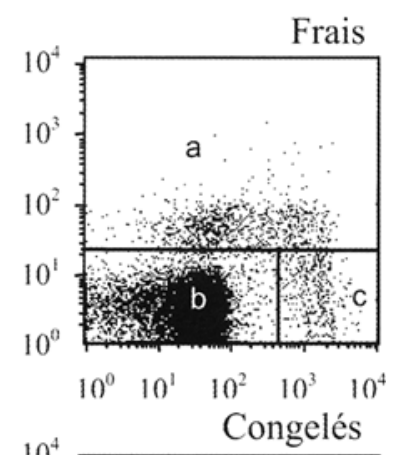

IP

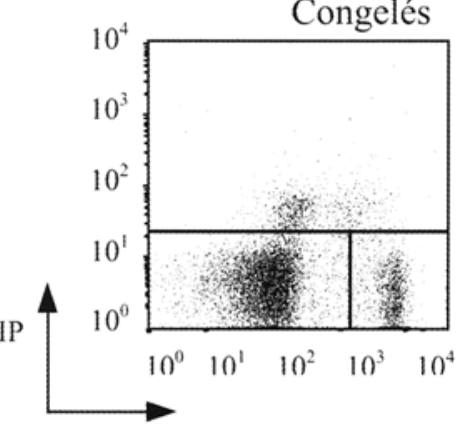

PNA-FITC

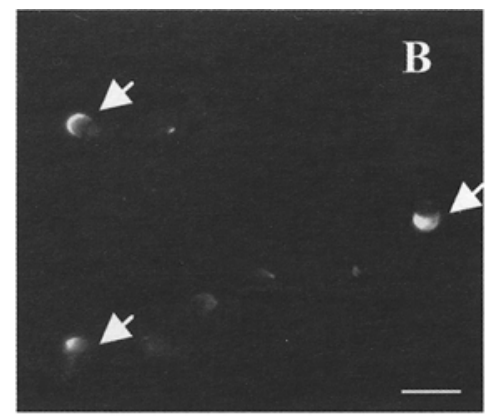

C

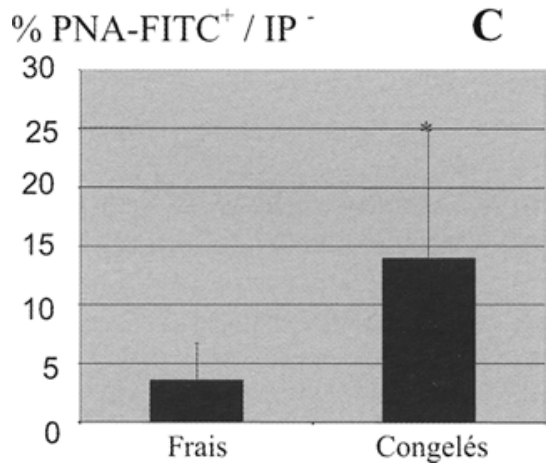

Figure 5 : Etude la réaction acrosomique.

$A$ : Cytogrammes PNA-FITC/IP. a représente les spermatozoïdes morts $(I P+)$, b les spermatozoïdes avec un acrosome intact (PNAFITC-/IP-) et $c$ les spermatozoïdes avec un acrosome réagi (PNA-FITC+/IP-).

$B$ : Localisation du marquage PNA-FITC dans des spermatozoïdes congelés, échelle $=5 \mu M$.

C: Proportion de spermatozoïdes vivants avec un acrosome réagi (PNA+/IP-) avant et après congélation. * signifie que les 2 moyennes sont différentes avec $p<0,05$. 
auparavant [10], nous confirmons que la congélation/ décongélation des spermatozoïdes bovins induit l'apparition d'un processus ressemblant à l'apoptose.

La congélation/décongélation est responsable de la diminution massive de la proportion de spermatozoïdes bovins avec un $\Delta \psi^{m}$ faible. Cette diminution du $\Delta \psi_{\text {mpeut entraîner }}$ une dysfonction mitochondriale, empêchant le renouvellement de l'ATP. Ainsi, un manque d'énergie du à une diminution de la quantité en ATP pourrait être responsable de la diminution de la mobilité observée après congélation/ décongélation [12]. Pendant l'apoptose, le relargage des facteurs pro-apoptotiques dans le cytoplasme peut être une autre conséquence de la perte d'intégrité membranaire des mitochondries. Si les spermatozoïdes contiennent ces pro facteurs, la diminution du $\Delta \psi^{m}$ pourrait être responsable de l'entrée dans le cytoplasme et/ou de l'activation des facteurs proapoptotiques.

Nous avons déterminé que l'AIF était présent dans les spermatozoïdes bovins. L'AIF est un facteur pro-apoptotique qui est normalement localisé au niveau de la mitochondrie et qui est relargué dans le cytoplasme des cellules apoptotiques [7]. La présence d'AlF dans les spermatozoïdes bovins suggère la possibilité que l'AIF soit relargué des mitochondries au cours de la congélation. II serait ainsi intéressant de préciser les différentes localisations de l'AIF au cours du processus de congélation.

La congélation/décongélation des spermatozoïdes bovins induit l'activation des caspases. De plus, nous avons localisé par immunofluorescence les caspases actives au niveau de la pièce intermédiaire, ce qui correspond à la localisation des mitochondries dans le spermatozoïde. Ceci donne des éléments pour considérer la pièce intermédiaire comme une région spécialisée pour l'apoptose. Cependant, le rôle joué par les caspases dans le spermatozoïde reste encore à élucider. Même si la plupart des substrats des caspases sont des protéines impliquées dans l'apoptose, ils peuvent être aussi impliqués dans d'autres processus biologiques tels que le cycle cellulaire et la différenciation [4]. Afin de mieux comprendre le rôle de cette activation dans le spermatozoïde, il serait intéressant de spécifier par western blot quelles sont les caspases activées par la congélation. Par ailleurs, nous avons observé qu'après congélation/décongélation, une large majorité des spermatozoïdes vivants présente un $\Delta \psi^{m}$ faible tandis que seulement quelques spermatozoïdes vivants contiennent des caspases actives. Ces résultats suggèrent la possible implication d'une voie indépendante des caspases. Par exemple, I'AIF, que nous avons mis en évidence dans les spermatozoïdes, pourrait jouer ce rôle.

L'analyse de la perméabilité au Yo-Pro-1 donne des informations sur les modifications membranaires relatives à l'apoptose [5]. La congélation/décongélation des spermatozoïdes bovins induit l'apparition d'une population de cellules vivantes mais perméables au Yo-Pro-1. Ce résultat est cohérent avec l'effet de la congélation sur les membranes [12]. Par ailleurs nous avons confirmé en cytométrie en flux que la congélation des spermatozoïdes induisait la réaction acrosomique. II serait intéressant de déterminer si ce sont les spermatozoïdes présentant un profil apoptotique qui possèdent également un acrosome réagi.

Nous avons mis en évidence que les marqueurs apoptotiques apparaissent à des moments différents du processus de congélation. Après dilution dans le milieu de congélation, on observe une diminution du $\Delta \psi^{m}$; après équilibration, une activation des caspases ; et après le processus complet de congélation/décongélation, une augmentation de la perméabilité membranaire. Ce résultat suggère que ce n'est pas seulement la formation de cristaux de glace qui est à l'origine des différentes modifications cellulaires dues à la congélation/décongélation [6]. Les caractéristiques de l'apoptose dans le spermatozoïde apparaissent selon la même cinétique qu'au cours de l'apoptose classique $[1,8$, $14,19]$. Afin de déterminer si elles appartiennent au même processus, il serait intéressant de déterminer si elles dépendent les unes des autres.

Une meilleure compréhension des mécanismes induits par la congélation devrait fournir des informations précieuses dans le but d'améliorer la congélation des spermatozoïdes.

\section{REFERENCES}

1. BRATTON D.L., FADOK V.A., RICHTER D.A., KAILEY J.M., GUTHRIE L.A., HENSON P.M. : Appearance of phosphatidylserine on apoptotic cells requires calcium-mediated nonspecific flip-flop and is enhanced by loss of the aminophospholipid translocase. J. Biol. Chem., 1997, 272 : 26159-26165.

2. CASTEDO M., FERRI K., ROUMIER T., METIVIER D., ZAMZAMI N., KROEMER G. : Quantitation of mitochondrial alterations associated with apoptosis. J. Immunol. Methods, 2002, $265: 39-47$.

3. DUVAL R., BELLET V., DELEBASSEE S., BOSGIRAUD C. : Implication of caspases during maedi-visna virus-induced apoptosis. J. Gen. Virol., 2002, 83 : 3153-3161.

4 FISCHER U., JANICKE R.U., SCHULZE-OSTHOFF K. : Many cuts to ruin : a comprehensive update of caspase substrates. Cell Death Differ., 2003, $10: 76-100$.

5. IDZIOREK T., ESTAQUIER J., DE BELS F., AMEISEN J.C. : YOPRO-1 permits cytofluorometric analysis of programmed cell death (apoptosis) without interfering with cell viability. J. Immunol. Methods, 1995, 185 : 249-258.

6. ISACHENKO E., ISACHENKO V., KATKOV, II, DESSOLE S., NAWROTH F. : Vitrification of mammalian spermatozoa in the absence of cryoprotectants: from past practical difficulties to present success. Reprod. Biomed. Online, 2003, $6: 191-200$.

7. JOZAN., SUSIN S.A., DAUGAS E. et al. : Essential role of the mitochondrial apoptosis-inducing factor in programmed cell death. Nature, 2001, $410: 549-554$.

8. KERR J.F., WYLLIE A.H., CURRIE A.R. : Apoptosis: a basic biological phenomenon with wide-ranging implications in tissue kinetics. Br. J. Cancer, 1972, 26 : 239-257.

9. LUE Y.H., HIKIM A.P., SWERDLOFF R.S. et al. : Single exposure to heat induces stage-specific germ cell apoptosis in rats : role of intratesticular testosterone on stage specificity. Endocrinology, 1999, $140: 1709-1717$.

10. MARTIN G., SABIDO O., DURAND P., LEVY R. Cryopreservation induces an apoptosis-like mechanism in bull sperm. Biol. Reprod., 2004, 71 : 28-37. 
11. MARTIN S.J., REUTELINGSPERGER C.P., MCGAHON A.J., et al. : Early redistribution of plasma membrane phosphatidylserine is a general feature of apoptosis regardless of the initiating stimulus : inhibition by overexpression of $\mathrm{Bcl}-2$ and $\mathrm{Abl}$. J. Exp. Med., 1995, 182 : 1545-1556.

12. MEDEIROS C.M., FORELL F., OLIVEIRA A.T., RODRIGUES J.L. : Current status of sperm cryopreservation : why isn't it better ? Theriogenology, 2002, $57: 327-344$.

13. NAGY S., HALLAP T., JOHANNISSON A., RODRIGUEZ-MARTINEZ H. : Changes in plasma membrane and acrosome integrity of frozen-thawed bovine spermatozoa during a $4 \mathrm{~h}$ incubation as measured by multicolor flow cytometry. Anim. Reprod. Sci., 2004, 80 : 225-235.

14. RAVAGNAN L., ROUMIER T., KROEMER G. : Mitochondria, the killer organelles and their weapons. J. Cell. Physiol., 2002, 192 : 131-137.

15. SAKKAS D., MARIETHOZ E., ST JOHN J.C. : Abnormal sperm parameters in humans are indicative of an abortive apoptotic mechanism linked to the Fas-mediated pathway. Exp. Cell. Res., 1999, 251 : 350-355.

16. SHRIVASTAVA P., SODHI A., RANJAN P. : Anticancer druginduced apoptosis in human monocytic leukemic cell line U937 requires activation of endonuclease(s). Anticancer Drugs, 2000, 11 : 39-48.

17. VAN WAGTENDONK-DE LEEUW A.M., HARING R.M., KAALLANSBERGEN L.M., DEN DAAS J.H. : Fertility results using bovine semen cryopreserved with extenders based on egg yolk and soy bean extract. Theriogenology, 2000, $54: 57-67$.

18. VISHWANATH R., SHANNON P. : Storage of bovine semen in liquid and frozen state. Anim. Reprod. Sci., 2000, 62 : 23-53.

19. WYLLIE A.H., KERR J.F., CURRIE A.R. : Cell death: the significance of apoptosis. Int. Rev. Cytol., 1980, 68 : 251-306.

Poster sélectionné au $X X 1^{\circ}$ Congrès de la Société d'Andrologie de Langue Française, Clermont-Ferrand, 9-11 Décembre 2004.

Manuscrit reçu : mars 2005 ; accepté avril 2005.

\section{ABSTRACT}

Kinetics and characterisation of the "apoptosis-like" phenomenon induced during the cryopreservation process of bovine spermatozoa Guillaume MARTIN, Odile SABIDO, Philippe DURAND,
Rachel LEVY

Cryopreservation of ejaculated bovine spermatozoa induces severe cell death. In a preliminary study, we observed that cryopreservation and/or thawing was also associated with early apoptotic features in living spermatozoa: i) decrease of the mitochondrial membrane potential $\alpha \psi_{\mathrm{m}}$ ), ii) caspase activation, iii) increase of membrane permeability, without the appearance of late characteristics: iv) no DNA fragmentation. This process has been called "apoptosis-like".

In this study, we evaluated the consequences of each step of cryopreservation of bovine spermatozoa : dilution in cryopreservation medium, equilibration and cryopreservation in liquid nitrogen/thawing. Apoptosis and acrosomal reaction were analysed by flow cytometry and the presence of AIF (Apoptosis Inducing Factor) was analysed by western blot. We observed that dilution in cryopreservation medium induced a marked and immediate increase of the proportion of living spermatozoa with a low $\Delta \psi \mathrm{m}$. After equilibration, the proportion of living spermatozoa with active caspases then began to increase. After the complete cryopreservation/thawing process, this population reached a maximum, and a significant increase of membrane permeability was observed.

These results, showing that some features of the "apoptosis-like" phenomenon are initiated in the early steps of cryopreservation, suggest that ice formation may not be the only factor affecting spermatozoa. The consequence of this $\Delta \psi_{\mathbf{m}}$ decrease could be the release and/or activation of various pro-apoptotic factors in the cytoplasm. Presence of the pro-apoptotic AIF factor in bovine spermatozoa suggests a possible role of this protein during the cryopreservation process.

We also confirmed that cryopreservation of bovine spermatozoa induced an acrosomal reaction. It would be of interest to investigate the relationship between this acrosomal reaction and membrane permeability. A better understanding of the cellular mechanisms involved in sperm cryopreservation would help to improve the preservation of bovine sperm.

Key-words: apoptosis, bovine spermatozoa, cryopreservation, flow cytometry 